\title{
Spin Magnetic Resonance in Perspectives of Spin Science and Spin Technology Development
}

\author{
Kev M. Salikhov
}

Published online: 17 September 2014

(C) Springer-Verlag Wien 2014

This year we celebrate the anniversary of Applied Magnetic Resonance. In the Introduction to the first issue of the journal I wrote:

\begin{abstract}
"From my point of view there are two main considerations in favor of AMR. The methods of magnetic resonance are widely used in many fields of physics, chemistry, molecular biology, mineralogy etc. It may be said that they have become routine, but even in these fields the range of problems, which MR methods are used to solve is widening constantly. If one thinks of structural problems, MR methods are involved in the investigations of more and more complex structures (albumen, fractal structures, composite compounds, integral schemes etc.). Therefore, it is always necessary to develop new algorithms for solving problems by means of MR methods. These new algorithms may be described by specialists in the pages of a specialized journal "Applied Magnetic Resonance". This is even more important if one speaks about the application of MR methods in somewhat unusual fields of science, such as medicine, archaeology, ecology, agriculture etc. The impressive variety of applications of MR methods and their penetration into new fields of science and technology necessitate the appearance of a journal, targeted at this so as to stimulate the expansion of applications of MR.

No less important is the fact that this journal will bridge the gap between scientists from East and West. AMR, being practically the first scientific journal published in English in USSR, will help rapid communication of scientific results in the USSR to their colleagues abroad and vice versa. More important, in my opinion, is the fact that joint work within one journal will
\end{abstract}

This issue is the first of the two devoted to the 25-th anniversary of Applied Magnetic Resonance.

K. M. Salikhov $(\bowtie)$

Kazan Physical-Technical Institute, Russian Academy of Sciences, Kazan, Russia

e-mail: salikhov@kfti.knc.ru 
expand the international collaboration of scientists and will strengthen interdisciplinary bonds".

I believe that these reasonings are also actual nowadays. In past decades, the fields of magnetic resonance applications grew up very much and continue to develop further. Just to mention a few perspective applications of magnetic resonance methods one can refer to the dipolar pulse electron paramagnetic resonance spectroscopy for structure determination on the nanoscopic level, the nuclear magnetic resonance crystallography, the quantum computing with nuclear and electron spins as qubits using methodology of magnetic resonance spectroscopy to control their states, etc. Generally, nowadays magnetic resonance phenomenon is exploited not only to study properties of materials but it can also be used as a tool to control the spin states in technologies. Thus, applications of magnetic resonance methods have a flourishing future. I hope very much that Applied Magnetic Resonance will contribute to a successful development of magnetic resonance and its applications.

The anniversary of Applied Magnetic Resonance offers me a good opportunity to thank many friends and colleagues from the magnetic resonance community for their support by submitting nice manuscripts, reviewing manuscripts, and giving advice. In particular, I am very grateful to the members of all editorial and advisory boards for their efforts in keeping publications in Applied Magnetic Resonance on a high scientific level. It is my pleasure to send special thanks and congratulations to Prof. Ulrich Haeberlen (MPI Heidelberg), who celebrates his 80th birthday this year.

I am grateful to Dr. Laila V. Mosina for her long-term service to Applied Magnetic Resonance. In previous years Dr. Sergei M. Akhmin did a great contribution to production of the journal. I thank Igor A. Axenov for his design of the journal. Last but not least, I am grateful to colleagues from Springer for their efforts to make the journal successful.

Kev M. Salikhov

August 25, 2014 